\title{
POSITION SENSITIVITY IN GALLIUM ARSENIDE RADIATION DETECTORS
}

\author{
Ron Harper and Robert A. Hilko \\ EG\&G/Energy Measurements
}

Oral Presentation, also to appear in Conference Proceedings

I. Introduction

For several years, we have studied the electrical output of GaAs detectors in response to $\mathrm{MeV}$ protons. Beams from the Los Alamos National Laboratory's tandem Van de Graaff, bunched into pulses of about 0.7 -ns width, have been used to drive detectors into the current mode, and fast electronics have enabled characterization of the impulse response shapes and the absolute sensitivities.

Recently, we extended this line of investigation to measure output-charge spectra in response to low-current beams, in which the count rate was low and pulses due to individual ionizing particles were analyzed. The detectors studied were made of natural, monolithic GaAs that was neither doped nor radiation damaged. This material, known to have poor impulse-response, was chosen because of its large sensitivity, which enabled us to record signals above the noise. Two detectors were studied. One was a $1-\mathrm{mm}$ cube, biased on contacts applied to opposing faces such that the uniform electric field was perpendicular to the incident radiation. The other detector was an "interdigitated" design, in which a repeating pattern of conductive strips, $0.1-\mathrm{mm}$ wide and $0.3-\mathrm{mm}$ apart, applied voltage on the surface facing the beam.

\section{Experimental Method}

The first part of the work was the measurement of spectra of the output charge of the detectors when bombarded by a beam of $\mathrm{MeV}$-energy protons, which was collimated to a diameter of $0.1 \mathrm{~mm}$. A proton beam from the Los Alamos National Laboratory Ion Beam Facility's tandem Van de Graaff was scattered from a thin gold foil through the collimator, positioned at $45^{\circ}$ relative to the beam. The GaAs detector was mounted on a microadjustable stage just behind the collimator, so that the site of irradiation on the detector could be varied. Output pulses originating from the impacts of individual protons were preamplified with chargesensitive Lecroy 2004 preamplifiers, shaped with Lecroy 2011 amplifiers, and analyzed with a Lecroy 3500 multichannel analyzer.

The second part was the measurement of the time response of the detector to a $0.1 \mathrm{~mm}$ collimated bunched proton beam. The proton bunch width was less than $1 \mathrm{~ns}$, during which time many protons struck the detector, driving it into the current mode where individual proton impacts are unresolved. The details of this experimental technique are described in reference 1. 


\section{DISCLAIMER}

\section{Portions of this document may be illegible in electronic image products. Images are produced from the best available original document.}




\section{Results and Interpretation}

The first spectra taken with both detectors, with full-face proton irradiation, were complex. Suspecting a variation in sensitivity across the bias gap to be the cause, we collimated the beam to $0.1-\mathrm{mm}$ diameter and swept the beam position across the gaps. A pronounced position dependence was observed with the output being an order of magnitude larger near the negative electrodes than near the positive (see Figs. 1 and 2). We thought that the impulse response might also be different near opposite electrodes. So, we collimated the bunched beam and observed the current-mode response vs time as the site of irradiation varied. Near the positive electrode, quickly restoring pulses were generated. However, near the negative electrode large tails appeared on the output pulses (see Figs. 3 and 4). The tails persisted for over $0.5 \mu \mathrm{s}$ and dominated the net output charge, hence the signal in the time-unresolved data taken with the 0.5 -ms integrating preamplifier.

These results may be informative of charge carrier behavior in GaAs. Electrons have about 20 times the velocity of holes [2], so that their instantaneous induced current will be 20 times that due to holes, before trapping has eliminated any carriers. The lifetimes of holes and electrons are about the same, for equal $\mathrm{P}$ and $\mathrm{N}$ impurity concentrations [3], as is the case for this material, of sufficient purity to give electron mobility of $7600 \mathrm{~cm}^{2} / V s$.

We propose a model in which the quick part of the output pulse is due to the summed induced currents of electrons and holes until they are all trapped, and in which the tails are due to detrapped electrons. In this model, electrons ionized near the negative electrode first contribute to the quick signal, get trapped, then escape from the traps with some time constant, and appear to trickle out of the traps, contributing briefly to displacement current before getting retrapped.

This process repeats until they reach the positive electrode, which is a long way from the ionization site. We believe electrons, rather than holes, to be responsible for the tails because if it were holes going through cycles of trapping and detrapping, the tails would be present in detector output stimulated by irradiation near the positive electrode, and in fact none are seen there.

Lund and Olschner [4] have developed a Monte Carlo algorithm that will predict charge collection efficiency and output pulse shape as functions of electron and hole trapping and detrapping times. Their results qualitatively confirm our model, but searches of parameter space to fit our data have not been done.

A possible detector design is suggested by our results. In the past, GaAs time response has been improved by doping or radiation damaging, which introduces traps. The tails can be eliminated, but at the cost of a factor of a thousand in main peak sensitivity. It now appears that by masking off the region of the detector near the negative electrode, the tails can be eliminated with only a factor of about ten loss in peak gain. 


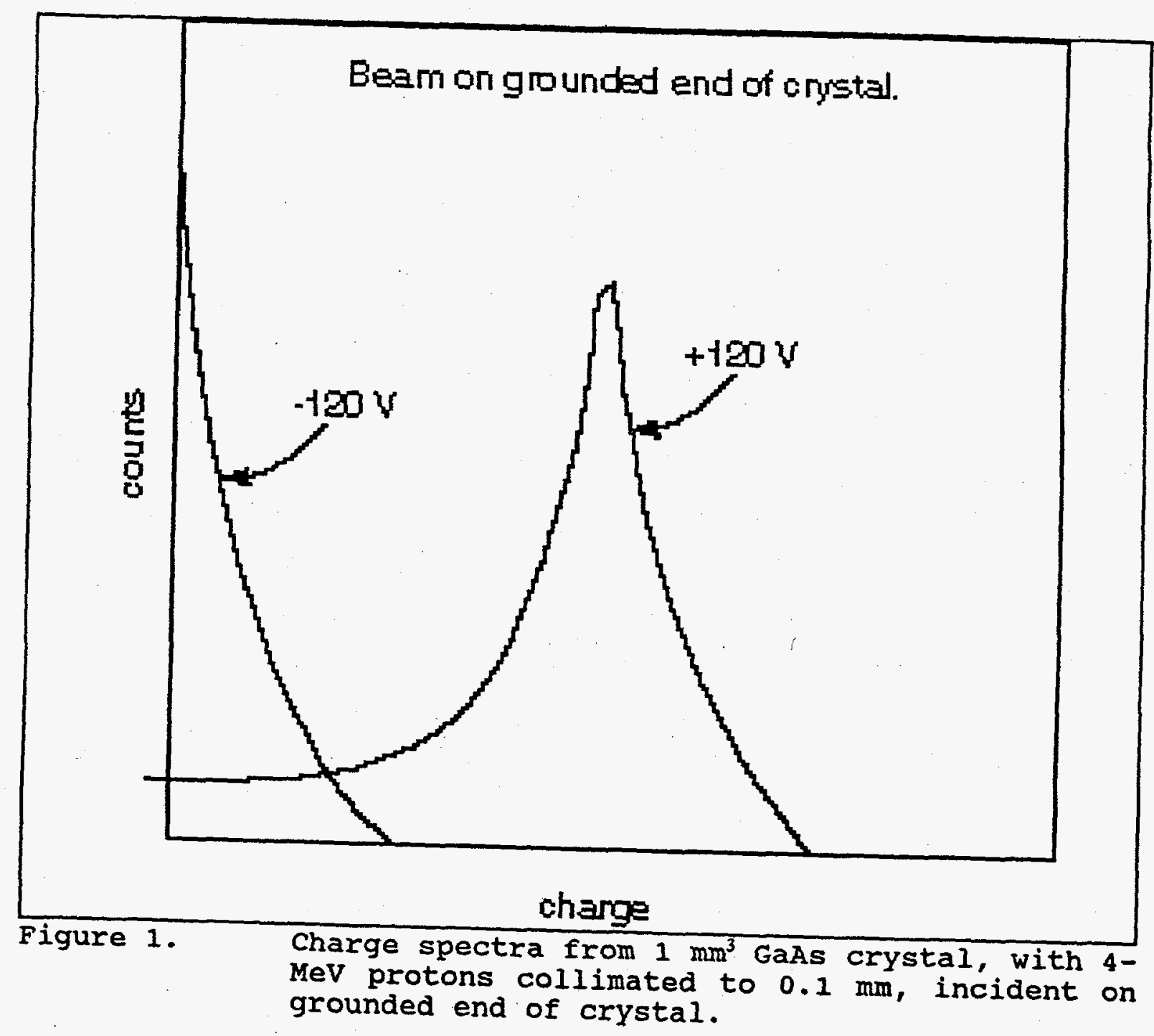

\section{DISCLAIMER}

This report was prepared as an account of work sponsored by an agency of the United States Government. Neither the United States Government nor any agency thereof, nor any of their employees, makes any warranty, express or implied, or assumes any legal liability or responsibility for the accuracy, completeness, or usefulness of any information, apparatus, product, or process disclosed, or represents that its use would not infringe privately owned rights. Reference herein to any specific commercial product, process, or service by trade name, trademark, manufacturer, or otherwise does not necessarily constitute or imply its endorsement, recommendation, or favoring by the United States Government or any agency thereof. The views and opinions of authors expressed herein do not necessarily state or reflect those of the United States Government or any agency thereof. 


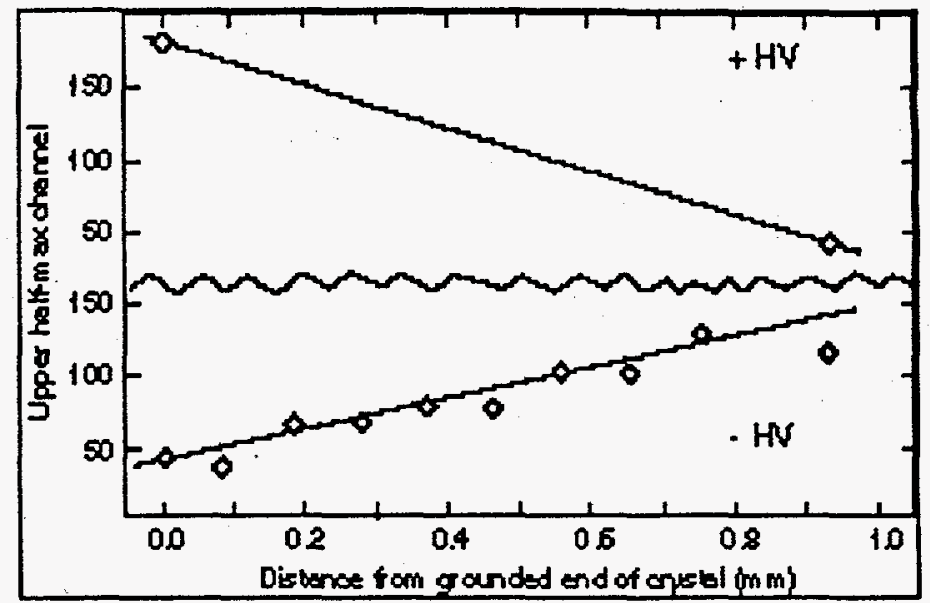

Figure 2. Upper half-maximum channel of charge spectra shown as functions of radiation site and bias polarity. 


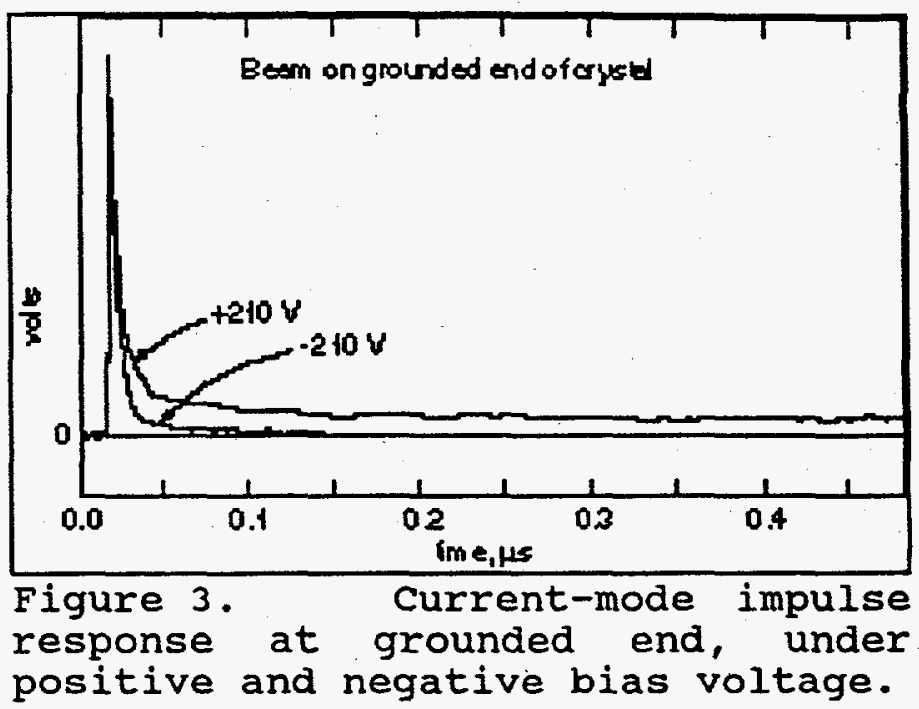




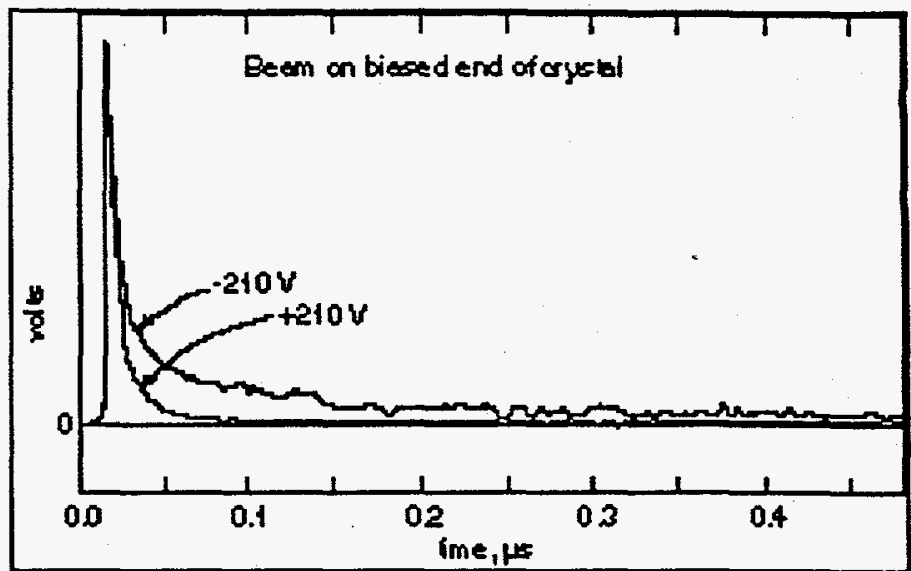

Figure 4. Current-mode impulse response at biased end, under positive and negative bias voltage. 
References:

[1] R. A. Hilko, R. W. Harper, et al., "Response of photoconductive detectors to low energy protons," Conference Record of the 1991 IEEE Nuclear Science Symposium, Vol. 1, November 1991.

[2] Properties of Gallium Arsenide. EMIS Datareviews Series No. 2. The Institute of Electrical Engineers.

[3] Gallium Arsenide: Materials, Devices, and Circuits. Ed. M. J. Howes and D.V. Morgan. 1985, John Wiley and Sons, Ltd. ISBN 0-471-90048-6.

[4] J. C. Lund and F. Olschner, NIM A288 (1990) 31-34. 